\title{
Immunoinformatics: Implications on Reverse Vaccinology
}

Mawadda Abd_Elraheem Awad_Elkareem*1 and Mohammed Ahmed Salih ${ }^{2}$

${ }^{1}$ School of pharmacy, Department of Biotechnology, Ahfad university for women, Omdurman, Khartoum ,Sudan

${ }^{2}$ School of pharmacy,Department of biotechnology, Africa city of technology, Khartoum, Sudan

\begin{abstract}
One area in which immunological studies have had most immediate and successful application is in the field of vaccination. Vaccines represent one of the greatest interventions in modern medicine [1]. Ever since Edward Jenner's first use of a vaccine against smallpox in 1796, the use of vaccines has become indispensable to the eradication of disease [2]. Then, Scientific progress has driven vaccine development from live attenuated and inactivated vaccines to purified recombinant one. The recent advances in bioinformatics, proteomics, immunoinformatics, structural biology and others have led to vaccinomics and reverse vaccinology as novel approaches for a generation of new vaccines [3]. Reverse vaccinology relies on the genomic information to identify relevant protein antigens and the design of algorithm for mapping potential $\mathrm{B}$ and $\mathrm{T}$ cell epitopes for diagnostic or vaccine purposes [4].
\end{abstract}

\section{Keywords: Vaccines; Immunological; Genome; Proteomics}

Immunoinformatics is scientific setting that targets the use of mathematical and computational approaches to comprehend, produce, process, and develop immunological data [5]. Since the 1980s, different immunoinformatic tools were developed and used aiming for vaccine design (Figure 1) [6].

As a result of sequencing human genome and other organism, huge amount of immunological data is accumulated. These wealthy data has been deposited in different database. The availability of different useful and user-friendly Web-based computational tools and searchable databases pave the way for predicting potential $\mathrm{B}, \mathrm{T}$ cell epitopes in protein sequences. The main objective when predicting these epitopes is the determination of the binding affinity of MHC molecules and antigenic peptides.

The tools in this field are based on statistical and machine learning system and are used for studying modeling molecular interactions (such as antigen processing and presentation) and also plays a role in defining new hypotheses related to understand the immune system mechanisms [7].

The concept of reverse vaccinology is based on selecting specific epitope of interest that are capable of provoking cellular as well as humoral immune response. The peptide candidate could be selected based on several criteria including, sequence conservancy, binding affinity to both MHC classes, allergenicity, etc.

Given the high cost and time requirements for new drugs development, vaccines remain as a viable alternative, but there too traditional techniques of live-attenuated or inactivated vaccines have the danger of allergenic reactions and others [3]. The developments in bioinformatics, proteomics, immunogenomics, structural biology and other sciences have spurred the growth of vaccinomics where computer assisted approaches serve to identify suitable peptide targets for eventual development of vaccines. These approaches reducing the workload of experimentalists with higher probability of success. Peptide vaccines have, over the last several years, begun to be looked on as more appropriate alternatives, which are economically affordable, require less time for development and hold the promise for reverse vaccinology $y^{3}$.

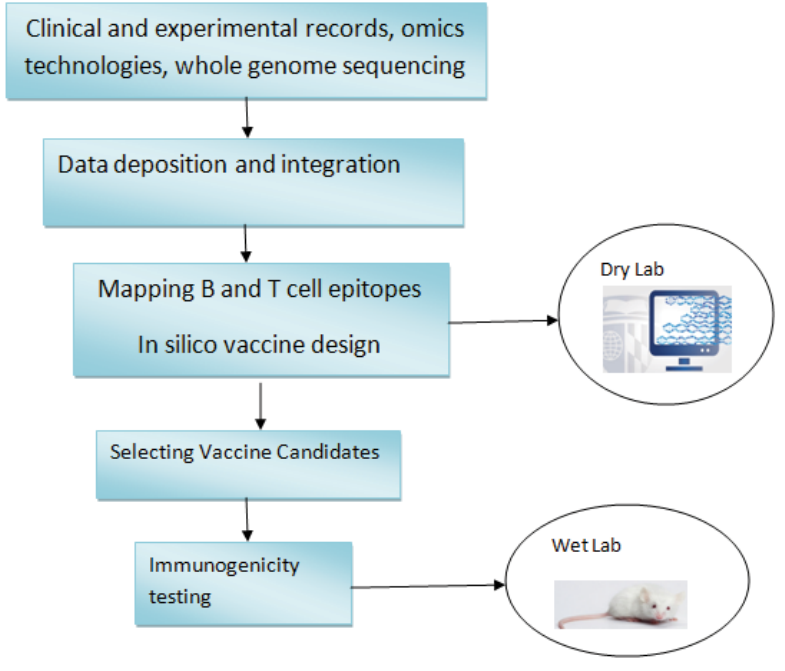

Figure 1: immunoinformatics implications on vaccine design.

\section{References}

1. Pulendran B, Ahmed R (2011) Immunological mechanisms of vaccination Nature Immunology 12: 509-517.

2. Nabel GJ (2013) Designing tomorrow's vaccines. New England Journal of Medicine 368: 551-560.

*Corresponding author: Mawadda Abd_Elraheem Awad_Elkareem, School of pharmacy, Department of Biotechnology, Ahfad university for women ,Omdurman, Khartoum ,Sudan, Tel: 00249917313523/00249116869326; E-mail: mawadda_ph@ hotmail.com

Received June 15, 2017; Accepted June 20, 2017; Published June 23, 2017

Citation: Awad_Elkareem MAE, Salih MA (2017) Immunoinformatics Implications on Reverse Vaccinology. Immunochem Immunopathol 3: 127. doi: 10.4172/24699756.1000127

Copyright: (c) 2017 Elneel MA. This is an open-access article distributed under the terms of the Creative Commons Attribution License, which permits unrestricted use, distribution, and reproduction in any medium, provided the original author and source are credited. 
Citation: Awad_Elkareem MAE, Salih MA (2017) Immunoinformatics Implications on Reverse Vaccinology. Immunochem Immunopathol 3: 127. doi: 10.4172/2469-9756.1000127

Page 2 of 2

3. Nandy A, Basak SC (2016) A Brief Review of Computer-Assisted Approaches to Rational Design of Peptide Vaccines. International Journal of Molecular Sciences 17: 666 .

4. Sette A, Rappuoli R (2010) Reverse vaccinology: developing vaccines in the era of genomics. Immunity 33: 530-541.

5. Khalili S, Jahangiri A, Borna H, Ahmadi ZK, Amani J (2014) Computational vaccinology and epitope vaccine design by immunoinformatics. Acta Microbiologica et Immunologica Hungarica 61: 285-307.

6. He Y, Rappuoli R, De Groot AS, Chen RT (2010) Emerging vaccine informatics. BioMed Research International.

7. Soria-Guerra RE, Nieto-Gomez R, Govea-Alonso DO, Rosales-Mendoza S (2015) An overview of bioinformatics tools for epitope prediction: implications on vaccine development. Journal of Biomedical Informatics 53: 405-414. 